\title{
Material properties determining
}

insecticidal activity of activated carbon on the pharaoh ant (Monomorium pharaonis)

Heleen Van Den Noortgate, Sreeprasanth Pulinthanathu Sree, Niels Ostyn, Bert Lagrain, Maarten Roeffaers, Tom Wenseleers, et al.

\section{Journal of Pest Science}

ISSN 1612-4758

J Pest Sci

DOI 10.1007/s10340-018-1024-7

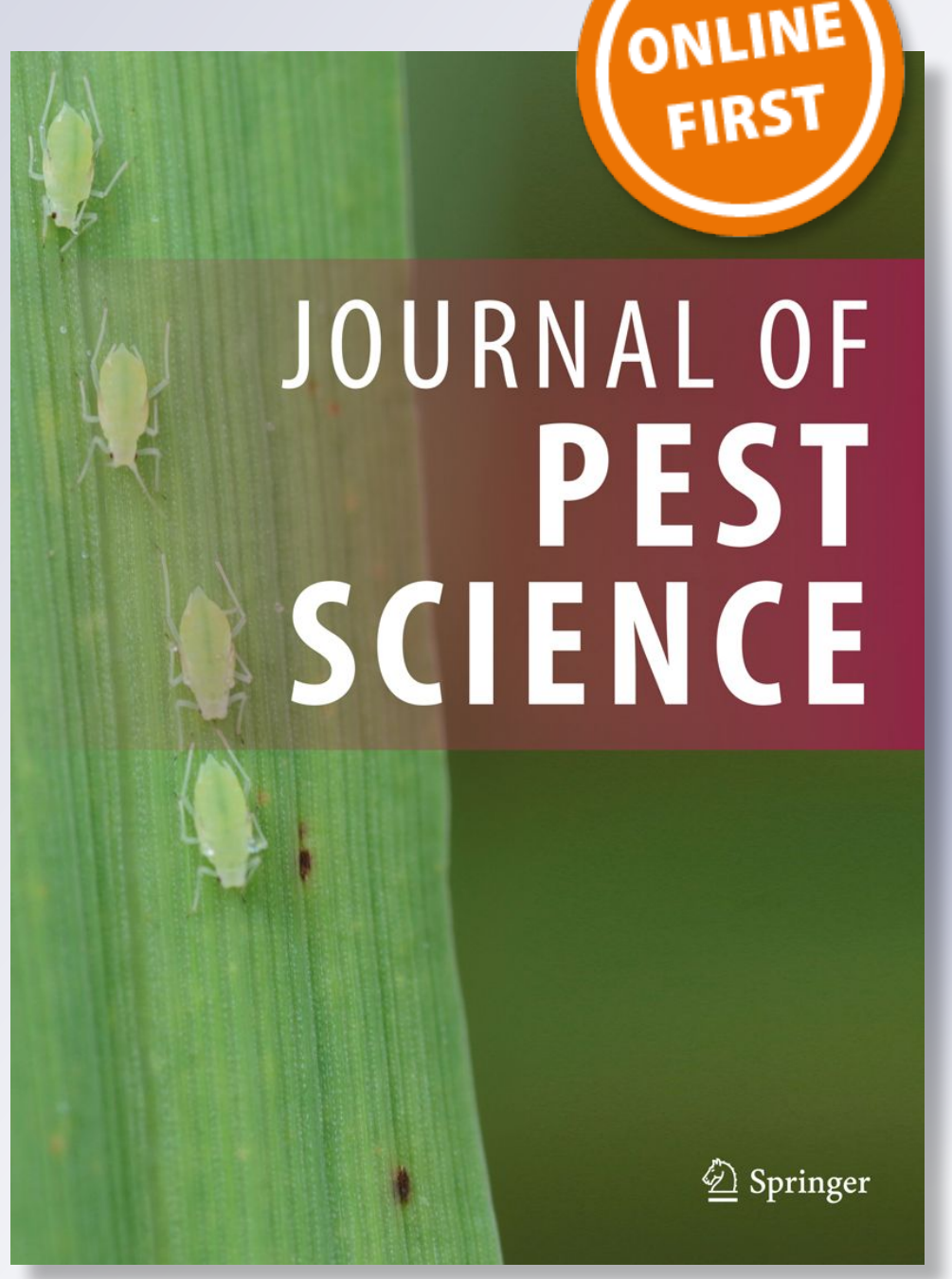

望 Springer 
Your article is protected by copyright and all rights are held exclusively by SpringerVerlag GmbH Germany, part of Springer Nature. This e-offprint is for personal use only and shall not be self-archived in electronic repositories. If you wish to self-archive your article, please use the accepted manuscript version for posting on your own website. You may further deposit the accepted manuscript version in any repository, provided it is only made publicly available 12 months after official publication or later and provided acknowledgement is given to the original source of publication and a link is inserted to the published article on Springer's website. The link must be accompanied by the following text: "The final publication is available at link.springer.com". 


\title{
Material properties determining insecticidal activity of activated carbon on the pharaoh ant (Monomorium pharaonis)
}

\author{
Heleen Van Den Noortgate ${ }^{1}\left[\right.$ ] Sreeprasanth Pulinthanathu Sree ${ }^{1}\left(\mathbb{D} \cdot\right.$ Niels Ostyn $^{1} \cdot$ Bert Lagrain $^{1}[$.

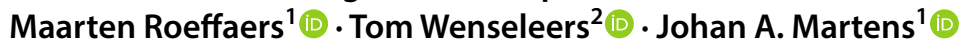

Received: 14 March 2018 / Revised: 18 June 2018 / Accepted: 13 July 2018

○) Springer-Verlag GmbH Germany, part of Springer Nature 2018

\begin{abstract}
Non-toxic and environmentally friendly insecticidal powders have grown in popularity as pest control products to substitute traditional pesticide-based methods. The pharaoh ant is an ideal test species for insecticidal dusts and it is an indoor pest, mostly found in heated buildings where it can pose severe risks because of its ability to destroy electrical equipment and transfer diseases in hospitals. The use of commercially available activated carbon and carbon black materials as insecticidal dust is of great interest, since these types of materials proved effective in previous research and they have been little researched in the past. In the present study, we compared the effectiveness of 11 different carbon materials to reveal the key material properties defining the insecticidal action. All of the materials, with the exception of Printex U, performed better than the benchmark diatomaceous earth, which is presently the most widely used insecticidal dust. The shortest median survival time, $25 \mathrm{~min}$, was observed upon exposure to activated carbon powder, which was significantly shorter than the 95 min observed for diatomaceous earth. Model selection revealed that particle size was the most important parameter determining insecticidal activity for activated carbon materials. Overall, our findings highlight the potential for the use of carbon materials as insecticidal agents, not just for the control of the pharaoh ant, but potentially also to control other domestic or agricultural pests in confined spaces, such as stored grain pests or bed bugs.
\end{abstract}

Keywords Pest management $\cdot$ Activated carbon $\cdot$ Desiccant powder $\cdot$ Pharaoh ant

\section{Key message}

- The pharaoh ant is a notorious and hard to manage pest.

- In previous research we found that carbon materials performed well against this pest.

- Thus far, little is known about their effectiveness and the important parameters influencing their effectiveness.

- In this study, the effectiveness of 18 different carbon samples against the pharaoh ant was studied.

Communicated by M. B. Isman.

Tom Wenseleers

Tom.Wenseleers@kuleuven.be

1 Centre for Surface Chemistry and Catalysis, KU Leuven, Celestijnenlaan 200F, Box 2461, Heverlee 3001, Belgium

2 Laboratory of Socio-ecology and Social Evolution, KU Leuven, Box 2461, Naamsestraat 59, Louvain 3000, Belgium
- The overall effectiveness of the materials was high, and the particle size was the most important parameter with powders and small pellets performing best.

\section{Introduction}

Diatomaceous earth is one of the oldest and most studied natural insecticidal powders (Akhtar and Isman 2016; Korunić 1997, 1998, 2013; Mewis and Ulrichs 2001; Quarles 1992) and has been shown to offer a nice alternative to traditional chemical-based pest control strategies, with fewer risks for the health of people, pets, and the environment. Such inert insecticidal dusts can make for a promising environmentally friendly and low hazard alternative to current state-of-the-art insecticides, with excellent safety if particle sizes do not fall into the category of inhalable dusts (Van Den Noortgate et al. 2017). So far, only few studies have tried to identify materials that might be more effective than diatomaceous 
earth-currently the most widely used inert insecticidal dusts (Ebeling and Wagner 1961a; Majumder 1962; Tarshis 1961) In a previous study, we already compared the insecticidal activity of various porous powders for their effectiveness against the pharaoh ant-a notorious domestic pest, for which chemical-based insecticides may pose obvious health and environmental risks, especially when the ants infest crowded buildings such as hospitals where they may spread infections and damage electrical equipment (Beatson 1972; Klotz et al. 2008). In that study, we found that many materials performed better than the diatomaceous earth benchmark material, especially zeolites, ordered mesoporous silica material, and carbon black (Van Den Noortgate et al. 2017). For the zeolites and ordered mesoporous silica materials, the most important parameters determining insecticidal activity were found to be the BET specific surface area and the area of the large mesopores (larger than $5 \mathrm{~nm}$ ). Finally, we also confirmed the uptake of epicuticular wax molecules in synthetic zeolites by GC-MS (gas chromatography-mass spectrometry) analysis, thus supporting the theory of Ebeling that the molecules are adsorbed by the insecticidal powders, which leads to the desiccation of the insects (Ebeling 1971; Ebeling and Wagner 1961a; Van Den Noortgate et al. 2017). The sampling of carbon materials in that study, however, was too limited to draw any conclusions on what material properties determine the insecticidal activity of that class of materials. In the present study, we therefore aimed to compare the effectiveness of 11 carbon materials as insecticides in the pharaoh ant and developed statistical models to test the critical material properties that explain their insecticidal activity.

Carbon materials already have many applications in the industry. Carbon black is widely used in printer toner and as a cheap filler in many products such as tires and plastics. Due to its electrical conductivity, it is also applied in the production of conductive packaging (Donnet et al. 1993; Pierson 1993). Activated carbon is of interest to electronic applications as supercapacitors (Fan et al. 2011; Hulicova-Jurcakova et al. 2009). It also has many applications as adsorbent, due to its high surface area and hydrophobicity. It is widely used as a filter material for liquids (Li et al. 2002) and gasses and even recommended as an emergency solution to intoxication, because it can remove toxins from the stomach by adsorption. Some other applications are related to fireworks and gunpowder as well as catalysts (Marsh 2006; Pierson 1993). Because of the wide commercial use, carbon materials are commercially available and rather inexpensive. The effectiveness of carbon as an inert insecticidal dust, however, has been little studied so far (Ebeling and Wagner 1961a; Majumder 1962; Majumder et al. 1959).

\section{Experimental methods}

\section{Pharaoh ant colonies}

A large colony of pharaoh ants was obtained from Purdue University (Indiana) and was further reared and split off into different subcolonies in a climate room, where they were kept at $26-28{ }^{\circ} \mathrm{C}$ and $60-70 \%$ relative humidity. Petri dishes coated with plaster were used as nesting space, and the ants were provided weekly with food (mealworms, grasshoppers, egg yolk, and sugar water). They were also continuously provided with fresh water.

\section{Test materials}

Samples of 11 different commercial carbon materials were tested for their insecticidal properties. An overview is provided in Table 1. Carbon black and activated carbon powders were used in the form at which they were commercially supplied. Since carbon materials are classified as nuisance dusts according to NIOSH standards and can thus be dangerous upon inhalation, we included materials with a larger grain size. Particles should be ideally $>100 \mu \mathrm{m}$ to no longer qualify as inhalable dust and pose health hazards (http://www.who.int/occupational_health/publications/en/ oehairbornedust3.pdf). Attempts to pelletize fine carbon powders to the desired particle size without the use of an adhesive, however, failed. Grinding of commercial pellets using pestle and mortar, by contrast, yielded excellent result and was found to be very effective to obtain different grain sizes. Subsequently, activated carbons were sieved into three different grain sizes: $250-500 \mu \mathrm{m}$ (large, $l$ ), 125-250 $\mu \mathrm{m}$ (medium, $m$ ), and $<125 \mu \mathrm{m}$ (small, $s$ ). The particle size was verified with scanning electron microscopy (SEM) (Fig. 1).

\section{Mortality tests}

To avoid the uptake of atmospheric water in the samples during the experiments, the materials were acclimatized in the climate room for $48 \mathrm{~h}$ before starting the survival assays. Subsequently, mortality tests were then conducted in cylindrical jars with a diameter of $3 \mathrm{~cm}$ and height of $7 \mathrm{~cm}$. To prevent the pharaoh ants from climbing the walls of the containers and escaping, the jars were coated with a commercial fluon (polytetrafluorethylene) coating. An abundant $50 \mathrm{mg}$ of powder was used per jar, which provided sufficient coverage of the container bottom. The containers with the powder were then left to acclimatize for another $48 \mathrm{~h}$ before 40 adult foragers from one of the colonies were added to the container, after which the survival assay was started. 
Table 1 Carbon material samples and suppliers

\begin{tabular}{llllll}
\hline Sample & Material & Original state & Supplier & $\begin{array}{l}\text { Commercial pellet } \\
\text { size }(\mathrm{mm})\end{array}$ & $\begin{array}{l}\text { Size after } \\
\text { fining } \\
(\mu \mathrm{m})\end{array}$ \\
\hline AC1 & MK & & & & NA \\
AC2 & AX-21 & Powder & Chemviron & NA & NA \\
AC3 & SAE super & Powder & Anderson & NA & NA \\
AC4s & F400 & Powder & Norit & NA & $<125$ \\
AC4m & F400 & Pellet & Chemviron & $1.14-4.75$ & $125-250$ \\
AC41 & F400 & Pellet & Chemviron & $1.14-4.75$ & $250-500$ \\
AC5 & BL & Pellet & Chemviron & $1.14-4.75$ & NA \\
AC6 & Cap super & Powder & Chemviron & NA & NA \\
AC7s & BGX & Powder & Norit & NA & $<125$ \\
AC7m & BGX & Pellet & CECA & $2.23-10.17$ & $125-250$ \\
AC71 & BGX & Pellet & CECA & $2.23-10.17$ & $250-500$ \\
CB1 & Vulcan XC72R & Pellet & CECA & $2.23-10.17$ & NA \\
AC8s & F12/470 & Pellet & Chemviron & $2.26-5.01$ & $<125$ \\
AC8m & F12/470 & Pellet & Chemviron & $2.26-5.01$ & $125-250$ \\
AC81 & F12/470 & Pellet & Chemviron & $2.26-5.01$ & $250-500$ \\
AC9s & AX-31 & Pellet & Anderson & $10.56(\mathrm{~d})$ & $<125$ \\
AC9m & AX-31 & Pellet & Anderson & $10.56(\mathrm{~d})$ & $125-250$ \\
AC91 & AX-31 & Pellet & Anderson & $10.56(\mathrm{~d})$ & $250-500$ \\
DE & Diatomaceous earth & Powder & Lumino & NA & NA \\
CB2 & Printex U & Powder & Degussa & NA & NA \\
\hline C & & Cabot & NA & \\
\hline
\end{tabular}

Commercial pellet size and size after fining are mentioned. For the commercial size, a range of sizes is mentioned for irregular pellets and for extruded pellets, the diameter $(d)$ of the pellets is mentioned (the length varies). For commercial products procured as powders, NA (not applicable) is used since they have no commercial pellet size and were not fined. Samples are named carbon black $(\mathrm{CB})$ and activated carbon (AC) followed by a unique number. The indices are for powders (no index), small particles $(s,<125 \mu \mathrm{m})$, medium particles $(m, 125-250 \mu \mathrm{m})$, and large particles $(l, 250-500 \mu \mathrm{m})$
Fig. 1 SEM images of carbon material F12/470. Left: ground to an average grain size of $200 \mu \mathrm{m}$ and right: very finely ground $(<125 \mu \mathrm{m})$

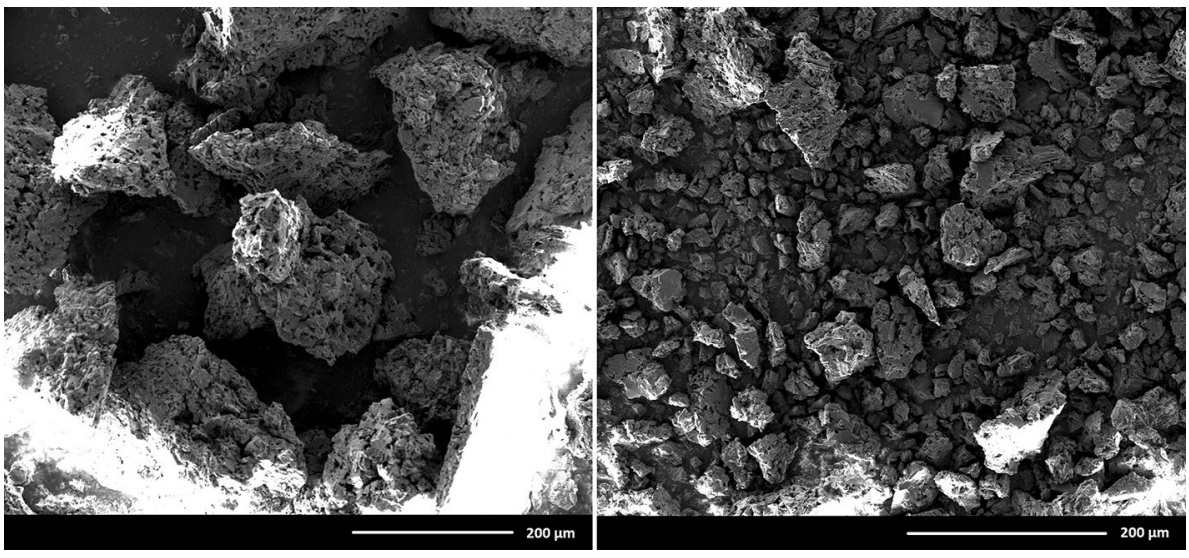

The assays were conducted in the climate room where the pharaoh ants were bred to avoid temperature and humidity fluctuations. Once the foragers were added to the test containers, they had no longer access to food and water. An empty jar (without powder) was used as reference. Every survival assay was measured in triplicate, and deaths (immobile and unresponsive foragers) were recorded every 5-10 min. The experiment was stopped when $100 \%$ mortality occurred, with the exception of the blank, which was monitored for $7 \mathrm{~h}$ every $30 \mathrm{~min}$. The blank was measured a total of nine times to check the reproducibility of the results. 


\section{Material properties analysis}

The carbon materials were characterized with respect to the following physical-chemical properties: BET surface area, DFT microporous and mesoporous surface area and BJH macroporous surface area, total pore volume, water adsorption capacity, particle size, and graphite content.

The porosity of the materials was characterized using nitrogen adsorption isotherms determined on a Quantachrome Autosorb-1 instrument. Samples were evacuated at $150{ }^{\circ} \mathrm{C}$ for $24 \mathrm{~h}$. Data were processed using AS1Win software. Brunauer-Emmett-Teller (BET) surface area was estimated in the $P / P_{0}$ range $0.01-0.3$ according to best linear fit; specific surface area in micropores (diameter $<2 \mathrm{~nm}$ ) and mesopores (diameters from 2 to $50 \mathrm{~nm}$ ) was estimated using (Quenched Solid) Diffusion Functional Theory ((QS) DFT). The mesopore surface area was divided into small mesopores $(<5 \mathrm{~nm})$ and large mesopores $(>5 \mathrm{~nm})$, a distinction which was found useful in previous work dealing with inorganic adsorbents containing different pore sizes (Van Den Noortgate et al.). Nitrogen sorption analysis was performed on a Micromeritics Tristar at high relative pressures for determining the macroporous specific surface area. The specific surface area of macropores with diameters from 50 to $240 \mathrm{~nm}$ was obtained from Barrett-Joyner-Halenda (BJH) analysis.

The water adsorption capacity of the carbon materials was measured on a magnetic suspension balance (Robatherm) and used as a measure of hydrophilicity. The samples were evacuated at $150{ }^{\circ} \mathrm{C}$ for $24 \mathrm{~h}$. The samples were equilibrated at $23{ }^{\circ} \mathrm{C}$ in air with $72 \%$ relative humidity. The weight gain owing to water adsorption divided by the pore volume of the material was used to quantify hydrophilicity. In the literature, similar methods using oil instead of water have been used to characterize the affinity of the adsorbent (Ebeling and Wagner 1961a, b; Korunić 2013; Majumder 1962).

The particle size was measured using scanning electron microscopy (Nova NanoSEM450, FEI). To this end, the samples were loaded onto a piece of carbon tape on an aluminum stub and observed at low voltages $(0.5-1 \mathrm{kV})$ to avoid charging of the material (Fig. 1).

Graphite content was measured based on obtained Raman spectra, which were recorded on a TriVistaTM spectrometer system with three action series dispersion stages and a charge-coupled device detector (Princeton Instruments) maintained at $-120{ }^{\circ} \mathrm{C}$. The laser source consisted of a coherent argon laser (300C MotoFreDTM, Innova ${ }^{\circledR}$ technology). The carbon samples were analyzed using a green laser (wavelength $514 \mathrm{~nm}, 2-7 \mathrm{~mW}$ ). Trivista software (VistaControl V4.0) was used to calibrate the system, and spectra were measured in the range of $50-4000 \mathrm{~cm}^{-1}$ wave numbers using frame exposure times of 1-5 min. These spectra were analyzed in Igor Pro (version 6.36) using the best peak fit (Lorentzian) and baseline fit (linear). Subsequently, the intensity of the $\mathrm{D}$ (Id) band over the intensity of the $\mathrm{G}$ band (Ig) was obtained and used as a measurement of the graphite versus amorphous carbon content of the materials.

\section{Statistical analysis}

The Cox proportional hazards model (coxph) was used to compare the survival rates of the different carbon samples. To this end, the survival package in $\mathrm{R}$ version 3.2.3 was used. Different replicates were included as a cluster factor into the models, and material type was included as a fixed factor. The difference in survival between the samples and the blank was calculated using Dunnett's post hoc tests using the lsmeans package. The data were visualized by plotting the Kaplan-Meier survival curves using the survfit function, and different colors were assigned according to their hazard value. The LT50 (median survival times) as well as the hazard ratios compared to the blank were also obtained from these curves. Subsequently, we used the bestglm package and an exhaustive search with leave-one-out cross-validation to determine which material properties were most important in predicting the insecticidal activity. To this extent, the $\log 10$-transformed hazard ratios were used as the dependent variable and eight material properties were included as possible covariates: BET specific surface area, micropore, small mesopore, large mesopore and macropore surface areas, water adsorption capacity, particle size, and the Raman structure parameter Id/Ig, which was a measure of the graphite content. The properties that were included in the model selection procedure were based on prior calculation of variance inflation factors, which enabled us to remove highly collinear variables such as the microporous pore volumes, which were highly correlated with the BET specific surface area. Models including both the Spmicro (microporous pore area) and the BET specific surface area also lead to high variance inflation factors $(>10)$, so for this reason they were also excluded. The predictive value of the models was measured based on their adjusted $R^{2}$ value, and the predictive value of each individual covariate was measured by the $\eta^{2}$ value, a measure of the partially explained variance of each covariate.

\section{Results}

\section{Mortality and material properties}

The different samples of activated carbon (AC) and carbon black (CB) materials used in the survival assays as well as their properties are listed in Table 2. Overall, the activated carbon samples are highly microporous materials with BET specific surface areas ranging from $884 \mathrm{~m}^{2} / \mathrm{g}$ for AC 8 to 


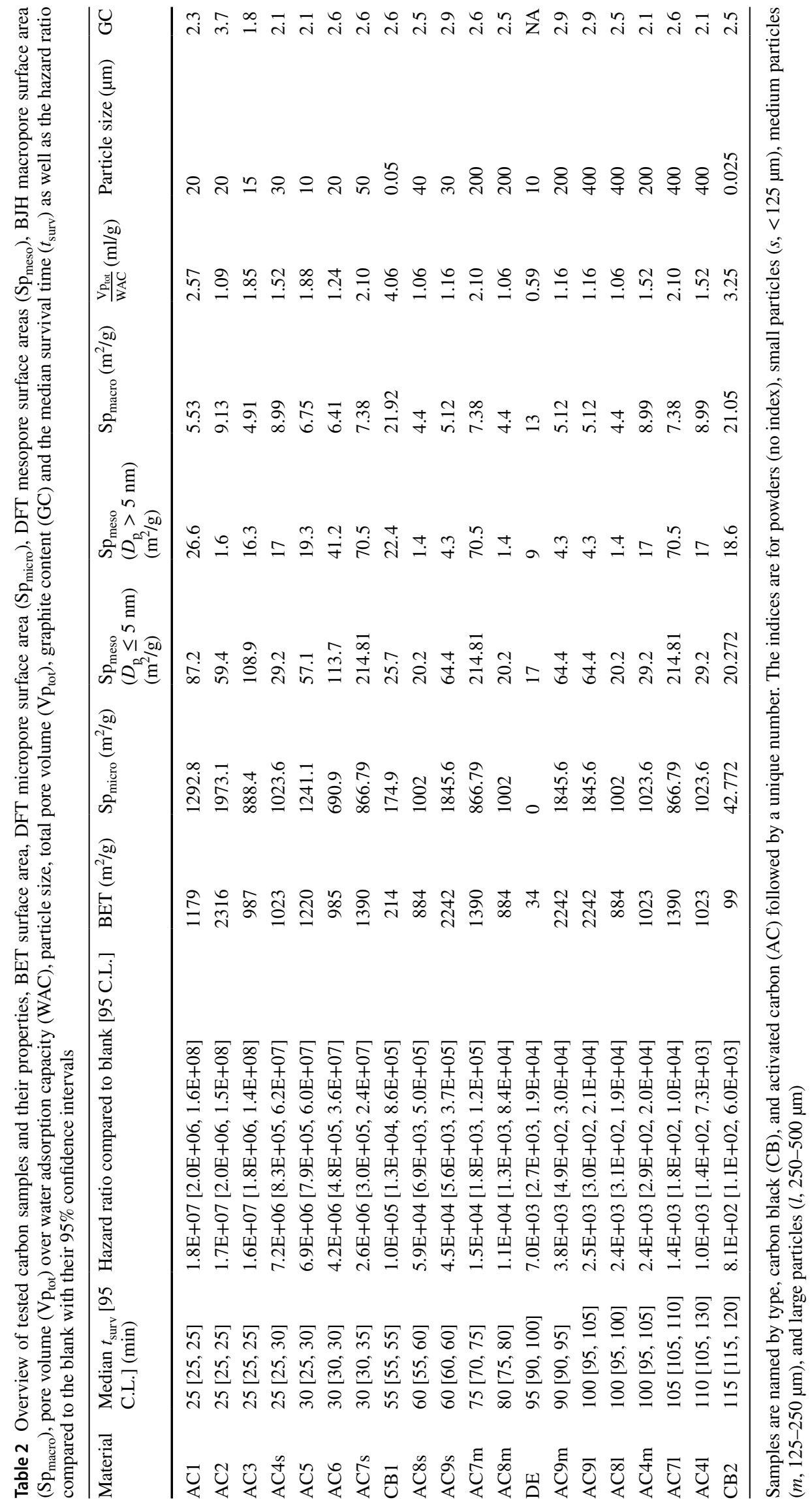


$2316 \mathrm{~m}^{2} / \mathrm{g}$ for AC2. They are quite hydrophilic with $V p_{\text {tot }}$ l water adsorption values of 1.06 for AC8 to 2.57 for $\mathrm{AC} 1$, but not as hydrophilic as diatomaceous earth with a value of 0.59. Particle sizes for the powders and smallest pellets range from $10 \mu \mathrm{m}$ (AC5) to $50 \mu \mathrm{m}$ (AC7), while the average particle size for the medium and large pellets is roughly 200 and $400 \mu \mathrm{m}$, respectively. This is as expected, since mediumsized pellets are distributed between 125 and $250 \mu \mathrm{m}$ while large pellets have sizes from 250 to $500 \mu \mathrm{m}$.

The carbon black samples are materials with a low porosity, with BET surface areas of 99 (CB2) and 214 (CB1). They are also less hydrophilic than the activated carbon materials resulting in $V p_{\text {tot }}$ /water adsorption values of, respectively, 3.25 and 4.06. Finally, their particle sizes are much smaller than in the activated carbon samples, with $0.025 \mu \mathrm{m}$ for $\mathrm{CB} 2$ and $0.05 \mu \mathrm{m}$ for CB1. The structure parameter $\mathrm{I}_{\mathrm{d}} / \mathrm{I}_{\mathrm{g}}$ ranges from 1.8 to 3.7 across all of the carbon samples used, while the carbon black samples both had values around 2.5 (Table 2).

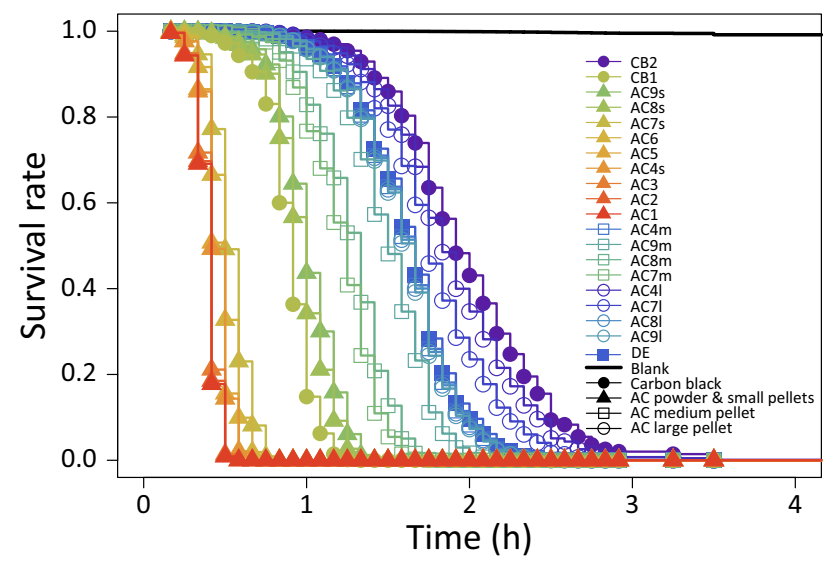

Fig. 2 pharaoh ant survival observed upon exposure to different carbon materials, illustrated using Kaplan-Meier survival curves which were based on fitted Cox proportional hazards models. Different symbols are used for the different types of carbon materials used and are ordered per type from least to most effective. Types are carbon black (CB) and activated carbon (AC). Activated carbon samples are further divided into powders (no index) and small particles ( $s$, $<125 \mu \mathrm{m})$, medium particles $(m, 125-250 \mu \mathrm{m})$, and large particles $(l, 250-500 \mu \mathrm{m})$. Diatomaceous earth (DE) was included as a benchmark material
The observed mortality of the pharaoh ants exposed to the different carbon materials is presented in Fig. 2 together with the observed mortality when they were exposed to the benchmark material diatomaceous earth. The carbon materials are divided into four categories: carbon black, activated carbon powders (and finely ground pellets), and activated carbon pellets broken to grain sizes of, respectively, 125-250 and $250-500 \mu \mathrm{m}$ (Table 1). In general, the activated carbon materials had shorter median survival times than the carbon black materials and the survival times seemed to increase with increasing particle size. The median survival times, shown in Table 2, ranged from 25 to $115 \mathrm{~min}$. Several materials, three powdered materials, and one smallest ground fraction of a pelletized material resulted in a median survival time of $25 \mathrm{~min}$. The longest survival time, $115 \mathrm{~min}$, was observed in pharaoh ants exposed to carbon black Printex U.

\section{Statistical analysis}

To determine the critical material properties explaining high insecticidal activity, we carried out an exhaustive model search to come to a model that maximized the explained variance in the measured $\log 10$-transformed hazard ratios, as measured by the adjusted $R^{2}$. This model search included the following covariates: BET, micropore, small mesopore, large mesopore and macropore surface areas, particle size, the ratio of pore volume over the amount of water adsorbed at $73 \%$ relative humidity, and the intensity of the D band over the intensity of the $\mathrm{G}$ band obtained from the Raman spectrum. While BET specific surface area is widely used for comparison of porous materials, it is often not very meaningful for microporous (carbon) materials (Barbieri et al. 2005; Thommes 2010; Thommes et al. 2015). Thus, both BET specific surface area and DFT measurements of the surface areas for different pore sizes were included in the properties. Béguin and Frackowiak (2010) already made this suggestion in 2010. As mentioned in Sect. 3.5, models including both BET and Spmicro (microporous DFT surface area) as covariates were excluded due to their high correlation.

The final best model, shown in Table 3, had an adjusted $R^{2}$ of 0.60 and contained the macropore surface area of the material and particle size as the critical most important material properties explaining insecticidal activity. Out of these, the particle size, however, was by far the

Table 3 List of properties of the optimal carbon material model: covariate coefficients, the tenth power, standard errors, $p$ values (significant $<0,05)$, the $t$ values from which the $p$ values were calculated and $\eta^{2}$, a measure of the explained variance for the covariates

\begin{tabular}{lcccccc}
\hline Term & Coefficient & $10^{\text {coefficient }}$ & SE & $t$ & $p$ & $\eta^{2}$ \\
\hline Intercept & 4.6816 & $48,037.418$ & 0.6104 & 7.67 & $9.5 \mathrm{E}-07$ \\
Spmacro & -0.1410 & 0.723 & 0.0540 & -2.61 & $1.9 \mathrm{E}-02$ \\
Particle size & -0.0091 & 0.979 & 0.0017 & -5.28 & $7.4 \mathrm{E}-05$ \\
\hline
\end{tabular}


Fig. 3 Partial effects of the retained critical material properties Spmacro (macropore surface area) and particle size (in $\mu \mathrm{m}$ ) on the measured insecticidal activity of 19 carbon materials in the pharaoh ant, as measured by the $\log 10$ of the hazard ratio calculated from a fitted Cox proportional hazards model, after an exhaustive best model search that included eight measured material properties $\left(\eta^{2}=0.15\right.$ and $0.62, p=1.9 \mathrm{E}-02$ and 7.4E-05)
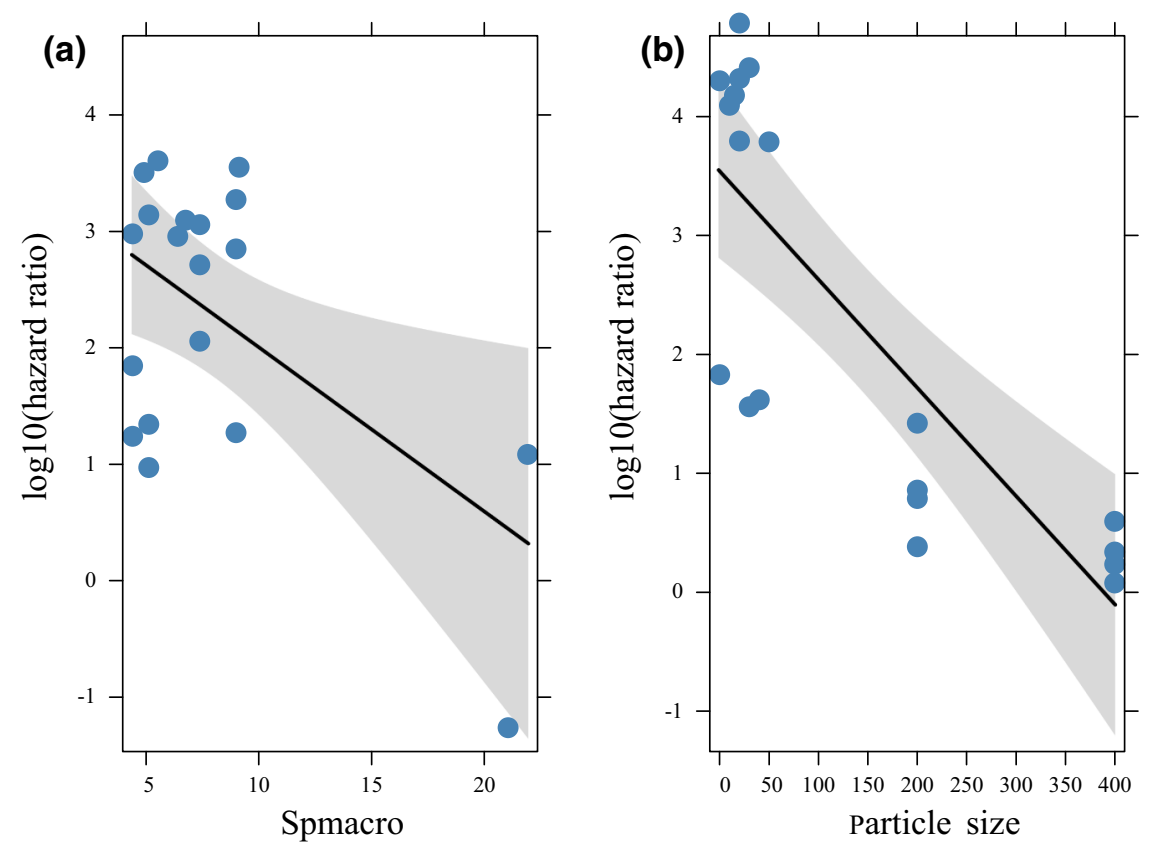

most important variable with an $\eta^{2}$ of 0.62 . Effect plots of both covariates were generated and are shown in Fig. 3. From these plots, it is clear that when the macroporous surface area becomes larger (as is the case for the carbon black samples) or when the particle size of the materials increases, the materials become less effective and the hazard ratio thus decreases.

Because of the severe effect on the model of adding the carbon black samples, it seemed prudent to recalculate the model for only the activated carbon materials. Since in general the carbon black materials have a much smaller particle size and are less microporous than activated carbon, including only one material type can reveal important parameters which otherwise can remain hidden. This generated a model including solely the particle size with an $R^{2}$ of 0.85 (Table 4 ). The particle size thus becomes very important (Fig. 4), which is to be expected as different particle sizes of the activated carbons were used and the effects thereof were already visible in the survival plot (Fig. 2).

\section{Discussion and conclusion}

Overall, our study shows that all tested carbon materials show great promise and were very effective in terms of their insecticidal activity against the pharaoh ant. The best median survival time of 25 min was even shorter than the best median survival time observed in the samples in our previous study, which primarily focused on zeolites and ordered mesoporous silica materials - 40 min for synthetic zeolite Y (Van Den Noortgate et al. 2017)—and nearly all measured median survival times were also shorter than the $95 \mathrm{~min}$ observed for the benchmark and currently most widely used material, diatomaceous earth. These results, obtained from samples which were not previously calcined and acclimatized to $70 \%$ relative humidity, indicate that carbon samples can also be effective at higher humidities. Indeed, there was also no clear influence of the water adsorption capacity, a measure of the hydrophilicity of the materials, on insecticidal activity. This conclusion contrasts with the findings of Majumder, who claimed that due to its hygroscopic properties, activated carbon in his research quickly lost its effectiveness at high humidities (Majumder 1962; Majumder et al. 1959). It also has to be noted that there are

Table 4 List of properties of the optimal activated carbon material model: covariate coefficients, the tenth power, standard errors, $p$ values (significant $<0.05$ ), the $t$ values from which the $p$ values were calculated and $\eta^{2}$, a measure of the explained variance

\begin{tabular}{lcllccc}
\hline Term & Coefficient & $10^{\text {coefficient }}$ & SE & $t$ & $p$ & $\eta^{2}$ \\
\hline Intercept & 0.5993 & 3.975 & 0.05387 & 11.1 & $1.2 \mathrm{E}-08$ \\
Particle size & -0.0024 & 0.994 & 0.00025 & -9.58 & $8.8 \mathrm{E}-08$ & 0.86 \\
\hline
\end{tabular}


Fig. 4 Effect of the critical factor particle size (in $\mu \mathrm{m}$ ) on the measured insecticidal activity of the 17 tested activated carbon materials in the pharaoh ant, as measured by the $\log 10$ of the hazard ratio calculated from a fitted Cox proportional hazards model, after an exhaustive best model search that included eight measured material properties $\left(R^{2}=0.85, p=8.8 \mathrm{E}-08\right)$

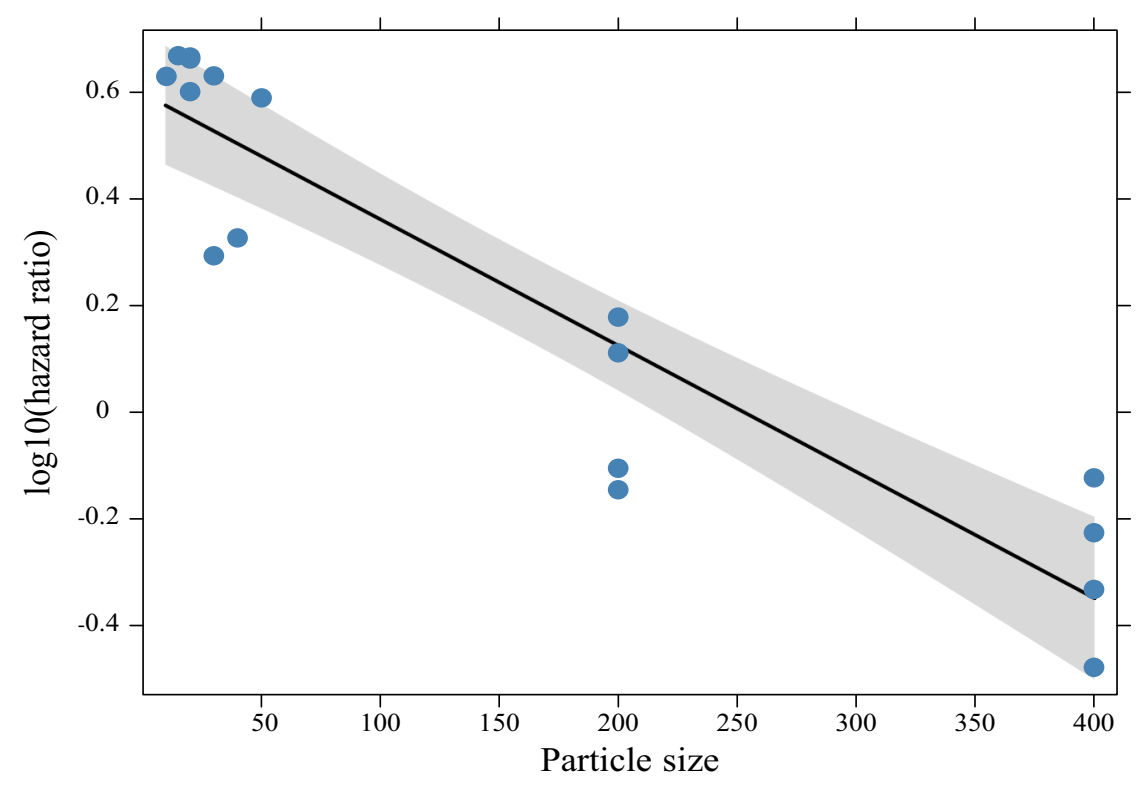

slight discrepancies between the BET surface area and the added total of the DFT surface areas (microporous, small mesoporous, and large mesoporous). These can be explained by the limited quantitative accuracy of the BET surface area calculation with respect to small pores in microporous materials (Thommes 2010; Thommes et al. 2015). However, neither BET nor DFT surface areas proved to be important predictive parameters.

Our results further show that activated carbon powders are in general more effective than the carbon black materials and that insecticidal activity is higher when smaller particle or grain sizes are used. These findings were also backed up by the results of the statistical analysis, which identified particle size as most important predictive parameter in the models. Remarkably, and with the exception of the $200 \mu \mathrm{m}$ grains of F400 and Printex U, all powdered materials as well as the $200 \mu \mathrm{m}$ grain size fractions of the materials all performed better than the diatomaceous earth benchmark. When the average grain size of the material was $400 \mu \mathrm{m}$ however, none of the materials performed any better than diatomaceous earth. Previously, it had been suggested that the mode of action of insecticidal dusts can be based either on passive adsorption of the protective waxy layer on the insect cuticle (Ebeling and Wagner 1961a), or on abrasion if materials with a high Mohs hardness value are used (David and Gardiner 1950). Our results show that also when soft materials like carbon materials are used, with a Mohs hardness value of only 2-3 (Patni et al. 2008), particle size remains the most important parameter. This is most likely due the higher adhesion linked to the smaller particle size (Katainen et al. 2006). Nevertheless, while carbon black materials have by far the smallest particle size, they are not very effective, probably due to their limited porosity and different porous properties from the activated carbon materials that limits their adsorptive properties. The different influence of the carbon materials versus the activated carbon materials is also clear from the statistical analysis, where the influence of the particle size is very prominent in the activated carbon samples, but offset by a negative influence of the macropore surface area when the carbon black samples are added to the dataset. Since these materials have a higher macroporous surface area than the activated carbon samples, a negative value attached to this covariate compensates for the smaller particle size, thereby resulting in a lower observed and predicted hazard value.

Overall, our study demonstrates that carbon materials are promising for use as insecticidal powders. Although our study used the pharaoh ant as a model, our conclusions may also extend to other domestic pests, such as the bed bug (Akhtar and Isman 2016) and some agricultural pests, such as stored grain pests (Mewis and Ulrichs 2001). Outdoor agricultural applications, however, may not be advised as this might also affect many nontarget, including beneficial, insects (Lockey 1988). For practical applications, the use of pellets is also recommended over the use of powders, as this would limit the danger and potential hazards of direct inhalation. Indeed, from our results, it is clear that even pellets (when sufficiently small, with an average particle size of ca. $200 \mu \mathrm{m}$ ) performed better than the benchmark material diatomaceous earth. The fact that carbon materials are very soft on the Mohs hardness scale and that they have a very low density may also have distinct advantages for the protection of stored grain pests, as the use of diatomaceous earth and derived materials there 
currently limits the flow of the grain processing and can cause wear in the machines that are used for grain processing (Fields and Korunic 2002). Carbon-based materials would be expected to cause less such problems, though this would require further study. One drawback of carbon materials when compared to diatomaceous earth is its color. The benchmark, white diatomaceous earth can be considered cleaner, since small quantities on light surfaces are hardly visible and black carbon can be considered, like soot, dirty. Some applications (in wall crevices) are not visible, and thus the problem does not exist there. It can also be tackled partially by using pellets instead of powders since the pelletized materials do not spread out easily and leave little residue on the surfaces they are used on, thus appearing more clean. The problem then remains solely for applications on soft (hard to clean) surfaces which people like to keep clean such as carpets (domestic pests), grain (stored product pests), and mattresses (bed bugs). To make the use of a black powder acceptable, the targeted area or object has to be sufficiently easily cleaned and the carbon material has to be significantly more effective than any white-colored powder fulfilling the same purpose.

Surprisingly, our results showed high effectiveness under a worst case scenario in real-life applications, which were high humidity and temperature. At these conditions, the powders can take up a lot of moisture from the environment, yet our results show that even then the materials we used retained high insecticidal activity. For domestic applications, humidity would always be lower than $70 \%$ and temperature would usually be lower than $25^{\circ} \mathrm{C}$ (ASHRAE Standard 55 of 1992), and such conditions would likely result in even higher mortality than observed in our test trials. On the other hand, in real-life conditions, the ants would have access to food and water and would have the possibility to evade the insecticidal powders, which would result in higher survival than observed in our in silico trials. A study aimed at optimizing the delivery method for real-life applications with measurements of induced mortality on whole ant colonies is currently under way.

\section{Author contributions}

JM, TW, BL conceived and designed research, HV executed experiments and wrote the manuscript, SPS conducted SEM measurements and MR and NO measured Raman spectra. All authors approved the manuscript.

Acknowledgements We would like to acknowledge the vzw Boterakker (Kinrooi, Belgium) and KU Leuven (Grant No.
INB-D1140-C32/16/020) for financial support. JAM acknowledges the Flemish Government for long-term structural funding (Methusalem).

\section{Compliance with ethical standards}

Conflict of interest All authors declare that they have no conflict of interest.

Ethical approval All applicable international, national, and/or institutional guidelines for the care and use of animals were followed. This article does not contain any studies with human participants performed by any of the authors.

\section{References}

Akhtar Y, Isman MB (2016) Efficacy of diatomaceous earth and a DE-aerosol formulation against the common bed bug, Cimex lectularius Linnaeus in the laboratory. J Pest Sci 89:1013-1021

Barbieri O, Hahn M, Herzog A, Kötz R (2005) Capacitance limits of high surface area activated carbons for double layer capacitors. Carbon 43:1303-1310

Beatson SH (1972) pharaoh's ants as pathogen vectors in hospitals. Lancet 1:425-427

Béguin F, Frackowiak E (2010) Carbons for electrochemical energy storage and conversion systems. CRC Press, Boca Raton

David WAL, Gardiner BOC (1950) Factors influencing the action of dust insecticides. Bull Entomol Res 41:1-61. https://doi. org/10.1017/S0007485300027474

Donnet J-B, Bansal RC, Wang M-J (1993) Carbon black, science and technology, 2nd edn. Marcel Dekker Inc, New York

Ebeling W (1971) Sorptive dusts for pest control. Annu Rev Entomol $16: 123-158$

Ebeling W, Wagner G (1961a) Physicochemical mechanisms for the removal of insect wax by means of finely divided powders. Hilgardia 30:531-564

Ebeling W, Wagner RE (1961b) Relation of lipid adsorptivity of powders to their suitability as insecticide diluents. Hilgardia 30:565-586

Fan Z, Yan J, Wei T, Zhi L, Ning G, Li T, Wei F (2011) Asymmetric supercapacitors based on graphene/ $\mathrm{MnO}_{2}$ and activated carbon nanofiber electrodes with high power and energy density. Adv Funct Mater 21:2366-2375. https://doi.org/10.1002/adfm.20110 0058

Fields P, Korunic Z (2002) Post-harvest insect control with inert dusts. In: Pimentel D (ed) Encyclopedia of pest management, vol 1. CRC Press, New York

Hulicova-Jurcakova D, Seredych M, Lu GQ, Bandosz TJ (2009) Combined effect of nitrogen- and oxygen-containing functional groups of microporous activated carbon on its electrochemical performance in supercapacitors. Adv Funct Mater 19:438-447. https:// doi.org/10.1002/adfm.200801236

Katainen J, Paajanen M, Ahtola E, Pore V, Lahtinen J (2006) Adhesion as an interplay between particle size and surface roughness. J Colloid Interface Sci 304:524-529

Klotz J, Hansen L, Pospischil R, Rust M (2008) Urban ants of North America and Europe: identification, biology and management. Cornell University Press, Ithaca

Korunić Z (1997) Rapid assessment of the insecticidal value of diatomaceous earths without conducting bioassays. J Stored Prod Res 33:219-229

Korunić Z (1998) Diatomaceous earths, a group of natural insecticides. J Stored Prod Res 34:87-97 
Korunić Z (2013) Diatomaceous earths-natural insecticides. Pestic Phytomed (Belgrade) 28:77-95

Li L, Quinlivan PA, Knappe DRU (2002) Effects of activated carbon surface chemistry and pore structure on the adsorption of organic contaminants from aqueous solution. Carbon 40:2085-2100

Lockey KH (1988) Lipids of the insect cuticle: origin, composition and function. Comp Biochem Physiol 89:595-645

Majumder SK (1962) Modes of insecticidal action of active carbon and clay on Tribolium castaneum (Hbst). Nature 193:1310-1311

Majumder SK, Narasimhan KS, Subrahmanyan V (1959) Insecticidal effects of activated charcoal and clays. Nature 184:1165-1166

Marsh HR-RF (2006) Activated carbon. Elsevier, Oxford

Mewis II, Ulrichs C (2001) Action of amorphous diatomaceous earth against different stages of the stored product pests Tribolium confusum, Tenebrio molitor, Sitophilus granarius and Plodia interpunctella. J Stored Prod Res 37:153-164

Patni AG, Ludlow DK, Adams CD (2008) Characteristics of ground granular activated carbon for rapid small-scale column tests. J Environ Eng 143:216-221

Pierson HO (1993) Handbook of carbon, graphite, diamond and fullerenes: properties, processing and applications. Noyes Publications, Park Ridge
Quarles W (1992) Diatomaceous earth for pest control. IPM Pract 14:1-11

Tarshis IB (1961) Laboratory and field studies with sorptive dusts for the control of arthropods affecting man and animal. Exp Parasit 11:10-33

Thommes M (2010) Physical adsorption characterization of nanoporous materials. Chem Ing Tech 82:1059-1073

Thommes M, Kaneko K, Neimark AV, Olivier JP, Rodriguez-Reinoso F, Rouquerol J, Sing KSW (2015) Physisorption of gases, with special reference to the evaluation of surface area and pore size distribution (IUPAC technical report). Pure Appl Chem 87:1052-1069

Van Den Noortgate H, Lagrain B, Sree SP, Kerkhofs S, Wenseleers T, Martens JA (2017) Material properties determining the insecticidal activity of highly divided porous materials on the pharaoh ant (Monomorium pharaonis). Pest Man Sci. https://doi. org/10.1002/ps.4814 